\title{
Decelerating molecules with microwave fields
}

\author{
Simon Merz, ${ }^{1}$ Nicolas Vanhaecke, ${ }^{1}$ Wolfgang Jäger, ${ }^{2}$ Melanie Schnell, $, 3,{ }^{3, *}$ and Gerard Meijer ${ }^{1, \dagger}$ \\ ${ }^{1}$ Fritz-Haber-Institut der Max-Planck-Gesellschaft, Faradayweg 4-6, 14195 Berlin, Germany \\ ${ }^{2}$ Department of Chemistry, University of Alberta, Edmonton, Alberta T6G 2G2, Canada \\ ${ }^{3}$ Center for Free-Electron Laser Science, Notkestrasse 85, 22607 Hamburg, Germany \\ ${ }^{4}$ Max-Planck-Institut für Kernphysik, Saupfercheckweg 1, 69117 Heidelberg, Germany
}

(Received 6 January 2012; published 19 June 2012)

\begin{abstract}
We report on the experimental realization of a microwave decelerator for neutral polar molecules, suitable for decelerating and focusing molecules in high-field-seeking states. The multistage decelerator consists of a cylindrical microwave cavity oscillating in the $\mathrm{TE}_{11 n}$ mode, with $n=12$ electric field maxima along the symmetry axis. By switching the microwave field on and off at the appropriate times, a beam of state-selected ammonia molecules with an incident mean velocity of $25 \mathrm{~m} / \mathrm{s}$ is guided while being spatially focused in the transverse direction and bunched in the forward direction. Deceleration from 20.0 to $16.9 \mathrm{~m} / \mathrm{s}$ and acceleration from 20.0 to $22.7 \mathrm{~m} / \mathrm{s}$ are demonstrated.
\end{abstract}

DOI: 10.1103/PhysRevA.85.063411

PACS number(s): $37.10 . \mathrm{Mn}, 37.20 .+\mathrm{j}, 33.20 . \mathrm{Bx}$

\section{INTRODUCTION}

The manipulation and control of beams of neutral polar molecules with electric and magnetic fields has enabled spectacular advances during the last decade and has rejuvenated the field of molecular beams [1,2]. Beams with a tunable velocity have been used to study (in)elastic scattering as a function of collision energy [3], slow molecular beams are being used to increase the resolution in spectroscopy experiments [4], and samples of trapped molecules have been used to measure lifetimes of long-lived metastable states [5], for instance. These advances have greatly benefited from the original development of Stark decelerators [6] and Zeeman decelerators [7] and their ongoing refinements.

The force on a polar molecule can be expressed as the product of an effective dipole moment and the gradient of the electric or magnetic field strength. This effective dipole moment depends on the quantum state of the molecule and is generally a function of the field strength. For the manipulation of molecules, a distinction has to be made between molecules in so-called low-field-seeking states and those in high-fieldseeking states. The electric and magnetic field strength can have a minimum in free space, and molecules in quantum states with a negative effective dipole moment, i.e., molecules in low-field-seeking states, can thus be stably trapped with static fields. By creating field minima on the molecular beam axis, these molecules can be readily confined around this axis, a feature that is exploited in multipole focusers as well as in the Stark and Zeeman decelerators mentioned above.

There is a strong interest in developing manipulation tools for molecules in high-field-seeking states, if only because the absolute ground state of any particle is high field seeking. As the Earnshaw theorem does not allow for a maximum of the field strength in free space using static fields alone, dynamic focusing (so-called alternating gradient) schemes have to be applied to confine molecules in high-field-seeking quantum states. The latter schemes are much like those that need to be

\footnotetext{
*melanie.schnell@asg.mpg.de

†meijer@fhi-berlin.mpg.de
}

applied to trap charged particles stably, and although guides [8-10], decelerators [11], and traps [12] for neutral molecules in high-field-seeking states have all been demonstrated by now, these approaches are experimentally more demanding, the confinement is less strong, and the trapping is intrinsically less stable.

Optical fields do provide a means to create electric field maxima in free space and are used in many laboratories to trap cold atoms as well as cold molecules that have been created from cold atoms via photo- or magnetoassociation [13]. Optical fields have also been successfully used to decelerate beams of polarizable molecules [14]. The electric field gradients that can be produced in an optical lattice formed by two counter-propagating pulsed laser beams are very large, leading to extremely rapid deceleration. The volume of the optical interaction region is, in general, rather small, however, and only a limited fraction of the molecules in the beam is accepted for the deceleration process. Moreover, it can be challenging to keep the temporal and spatial intensity profile of the laser pulses sufficiently well under control. To circumvent some of these problems, it has been proposed to use amplitude modulation of near-resonant infrared radiation inside a highfinesse cavity to construct an optical decelerator [15].

A promising alternative for the manipulation of the motion of molecules is the use of the microwave radiation field inside a resonant cavity, as the volume of the interaction region will be much larger and the control over the electric field distribution can be more precise. Using high-power microwave radiation, optical traps for polar molecules can in principle be made with a subkelvin depth [16], and a microwave decelerator, based on amplitude modulation of the standing-wave electric field in an open Fabry-Perot microwave cavity, has been proposed [17]. Thus far, only a deflector [18] and a lens [19] based on microwaves have been experimentally demonstrated for molecules.

We demonstrate here a microwave decelerator for neutral polar molecules. The multistage decelerator consists of a cylindrical microwave cavity oscillating in the $\mathrm{TE}_{11 n}$ mode, with $n=12$ electric field maxima along the symmetry axis. A pulsed beam of Stark-decelerated, state-selected ammonia $\left({ }^{14} \mathrm{NH}_{3}\right)$ molecules with an incident mean velocity of around 


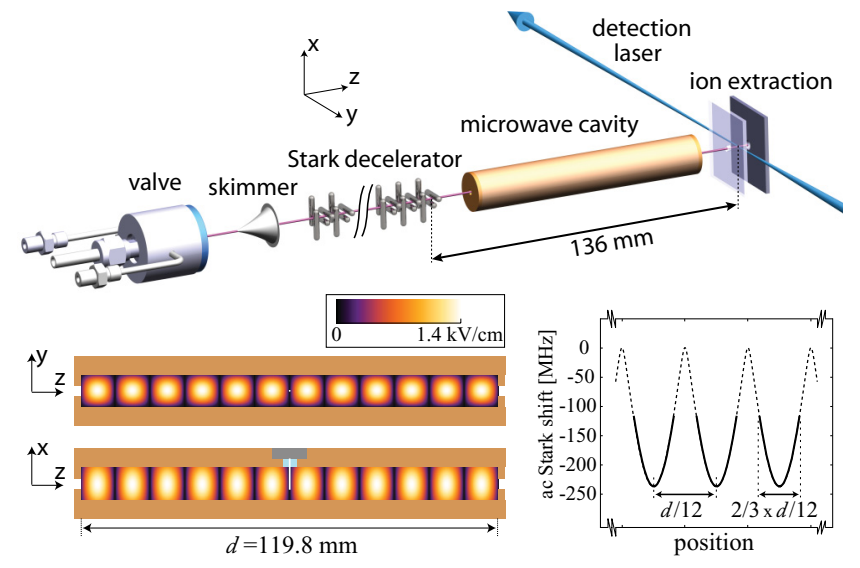

FIG. 1. (Color online) Upper panel: Schematic of the experimental setup. A 12-cm-long cylindrical microwave cavity is mounted behind a Stark decelerator. A packet of slow ammonia molecules, exiting the Stark decelerator, passes through this cavity and is then detected using resonant photoionization. Lower panel: False-color representation of the electric field distribution inside the microwave cavity when $3.0 \mathrm{~W}$ of microwave power at $\approx 23.7 \mathrm{GHz}$ is coupled to the $\mathrm{TE}_{1,1,12}$ mode. Cooling the copper cavity to liquid nitrogen temperatures results in a $Q$ factor of about 9150 and a maximum electric field strength on the axis of about $1.4 \mathrm{kV} / \mathrm{cm}$. The cavity is represented to scale, but for the sake of clarity the antenna is displayed five times thicker than in reality. The longitudinal potential wells experienced by the ammonia molecules on the axis of the cavity are shown in the lower right corner for a blue detuning of the microwave radiation of $58 \mathrm{MHz}$ from the molecular resonance. The portion of the potentials experienced by the synchronous molecule in guiding mode is represented in bold.

$20-25 \mathrm{~m} / \mathrm{s}$ is injected along the axis of the cavity. By switching the microwave field on and off at the appropriate times, the ammonia molecules are stably guided through the cavity and are focused, both transversely and longitudinally, into the detection region, directly behind the microwave cavity. Longitudinal spatial focusing ("bunching") of the ammonia beam in the detection region, as well as deceleration from 20.0 to $16.9 \mathrm{~m} / \mathrm{s}$ and acceleration from 20.0 to $22.7 \mathrm{~m} / \mathrm{s}$, is demonstrated.

\section{EXPERIMENTAL SETUP}

The experimental setup is shown schematically in Fig. 1. A detailed description of the molecular beam machine and, in particular, of the Stark deceleration of a beam of ammonia molecules is given elsewhere [20]. In the experiment, a single decelerated packet of $\mathrm{NH}_{3}$ molecules leaves the Stark decelerator in the upper inversion doublet component of the $|J, K\rangle=|1,1\rangle$ level, in the vibrational and electronic ground state. The mean longitudinal velocity of the molecules is set at around $20-25 \mathrm{~m} / \mathrm{s}$ and the full-width-at-half-maximum velocity spread is about $10 \mathrm{~m} / \mathrm{s}$. The decelerated packet leaving the decelerator contains about $10^{5}$ molecules and has a spatial extent of less than $2 \mathrm{~mm}$ along all directions. Here we define the time at which the Stark decelerator is switched off as $t=0$. At this time, the center of the packet of molecules is about $6 \mathrm{~mm}$ in front of the microwave cavity. The inner length of the microwave cavity is $d=119.8 \mathrm{~mm}$ (at room temperature), and there are identical 2-mm-thick end caps (with a 3-mm-diameter opening for the molecules) on either end. About $6 \mathrm{~mm}$ behind the microwave cavity the ammonia molecules are resonantly ionized between the extraction plates of a compact linear time-of-flight setup and the parent ion signal is recorded. The total distance that the molecules travel from $t=0$ up to the time of detection is thus about $136 \mathrm{~mm}$.

The cylindrical microwave cavity is made out of oxygenfree copper and has a precisely machined inner diameter of $9.57 \mathrm{~mm}$ (at room temperature). Microwave radiation around 23.7 GHz is coupled in via a 6.5-mm-long, 0.1-mm-diameter dipole antenna, located at the center of the cavity, to excite the $\mathrm{TE}_{1,1,12}$ mode. When the cavity is cooled down to $77 \mathrm{~K}$, this cavity mode is about $58 \mathrm{MHz}$ blue-shifted from the molecular resonance frequency $v_{0} \approx 23695 \mathrm{MHz}$, i.e., from the inversion transition of the $|J, K\rangle=|1,1\rangle$ level of the vibronic ground state of ${ }^{14} \mathrm{NH}_{3}$. From the observed width of the cavity resonance, a quality factor of the resonator of $Q=9150$ is deduced. Due to the blue detuning, molecules in the upper component of the inversion doublet, that are low field seeking in static electric fields, become high field seeking in the microwave field [19]. The radial dependence of the electric field in the microwave cavity, which is always strongest on the beam axis, thus results in a focusing of the ammonia molecules along this axis. This behavior was exploited in the demonstration of a microwave lens for ammonia molecules, for which low-order $\mathrm{TE}_{11 n}$ modes with $n=2$ or 4 were used [19].

The mode with $n=12$ electric field maxima on the symmetry axis that is used here creates electric field gradients in the longitudinal direction that are similar in magnitude to those in the transverse direction, as can be seen from the mode pattern shown in the lower left corner in Fig. 1. The longitudinal gradients can be used to influence and control the forward velocity of the molecules. When $3.0 \mathrm{~W}$ of microwave power is coupled to the cavity, the maximum electric field strength on the axis is around $1.4 \mathrm{kV} / \mathrm{cm}$, resulting in an ac Stark shift of approximately $250 \mathrm{MHz}$. As this is considerably larger than the detuning of $58 \mathrm{MHz}$, the Stark shift that the molecules experience near the field maxima scales almost linearly with the electric field strength [19]. This in turn implies that the longitudinal potential that the ammonia molecules experience is nearly perfectly harmonic around the position of each of the 12 electric field maxima, as shown explicitly in the lower right corner in Fig. 1.

\section{EXPERIMENTAL RESULTS}

Figure 2 shows the density of ammonia molecules behind the microwave cavity as a function of time. Without microwave radiation in the cavity [Fig. 2(a)], there is hardly any detectable signal; the slow, divergent beam of ammonia molecules is strongly diluted during free flight to the laser interaction region. When $3.0 \mathrm{~W}$ of microwaves is continuously coupled in [Fig. 2(b)], the cavity functions as a microwave lens and the ammonia molecules are transversally focused into the detection region. The observed broad arrival time distribution, centered around $5.2 \mathrm{~ms}$, reflects the broad initial velocity distribution of the molecules entering the microwave cavity. Figure 2(c) shows measurements recorded using a longitudinal 


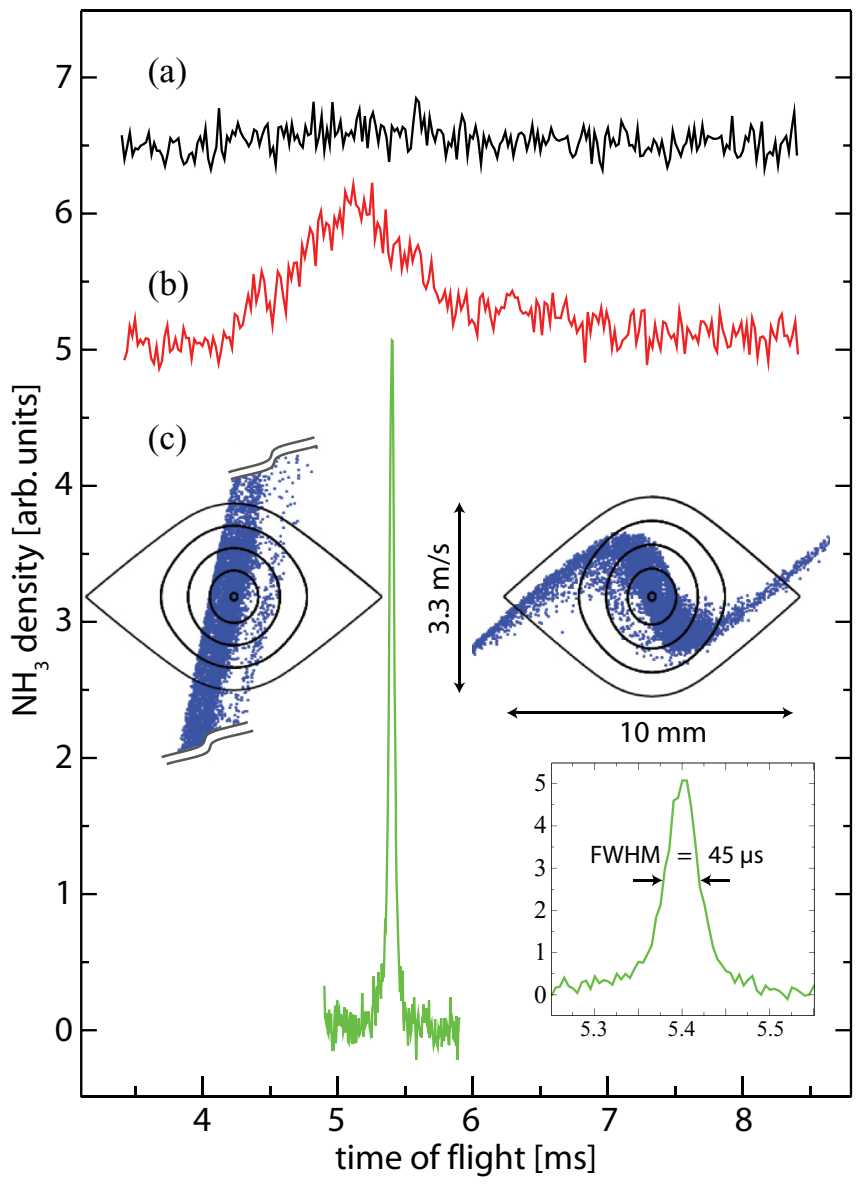

FIG. 2. (Color online) Density of ammonia molecules behind the microwave cavity as a function of time (in milliseconds) without microwaves (a), with $3.0 \mathrm{~W}$ of microwaves continuously coupled in (b), or with pulsed application of the microwaves for two-thirds of the time (c). Curves are offset for clarity, but all have the same vertical scale. For the measurements in (c), the microwaves are applied synchronously with the motion of an ammonia molecule that moves on the molecular beam axis at a forward velocity of $25 \mathrm{~m} / \mathrm{s}$. The longitudinal phase-space distribution of the molecules at the first time that the microwaves are switched on and at the last time that they are switched off is shown on the left and right side of the peak, respectively, superimposed on the longitudinal phase-space acceptance diagram.

guiding mode of operation. The microwaves are switched on and off every time a (fictitious) molecule that enters the cavity on the beam axis at a forward velocity of $v_{z}=25 \mathrm{~m} / \mathrm{s}$ reaches the position at which the electric field strength is $50 \%$ of its maximum value. This means that the microwave fields are on for a distance spanning two thirds of each electric field strength period $(2 / 3 \times d / 12 \approx 6.7 \mathrm{~mm})$, symmetrically spread around the electric field maxima, as shown schematically in the lower right corner in Fig. 1. Consequently, during its flight on the axis of the cavity, such a molecule, referred to as the synchronous molecule, only experiences the deepest part of the potential wells. The ammonia molecules are thus trapped in an effective traveling longitudinal potential well, much like the situation during guiding at a constant velocity in a Stark decelerator [21].

The observed arrival time distribution in Fig. 2(c) has an intense peak around $t=5.40 \mathrm{~ms}$, the expected arrival time for molecules being transported through the microwave cavity at a mean velocity of $25 \mathrm{~m} / \mathrm{s}$. This narrow peak is shown on an expanded time scale in the lower right corner in Fig. 2, from which it is seen to have a temporal width of $45 \mu \mathrm{s}$. This width mainly results from the length of the packet of molecules, although there might be a slight broadening due to the finite size of the (focused) ionization laser beam. Neglecting the latter effect sets the upper limit on the longitudinal size of the packet of ammonia molecules in the detection region to only $1.1 \mathrm{~mm}$. The packet is this compact because we selected the parameters so as to perform longitudinal spatial focusing in the detection region, i.e., a one-to-one image is made of the packet at the exit of the Stark decelerator in the detection region. The molecules contributing to this narrow peak would have given rise to a 10 times broader time-of-flight profile if the microwaves had been kept off or constantly on.

The principle of bunching is visualized in the insets in Fig. 2(c), where the calculated longitudinal phase-space distributions of the molecules near the entrance (left side) and close to the exit (right side) of the microwave cavity are shown, superimposed on the longitudinal phase-space acceptance of the microwave decelerator. Under the present operating conditions, the packet undergoes almost exactly half a rotation in phase space, such that a spatially narrow

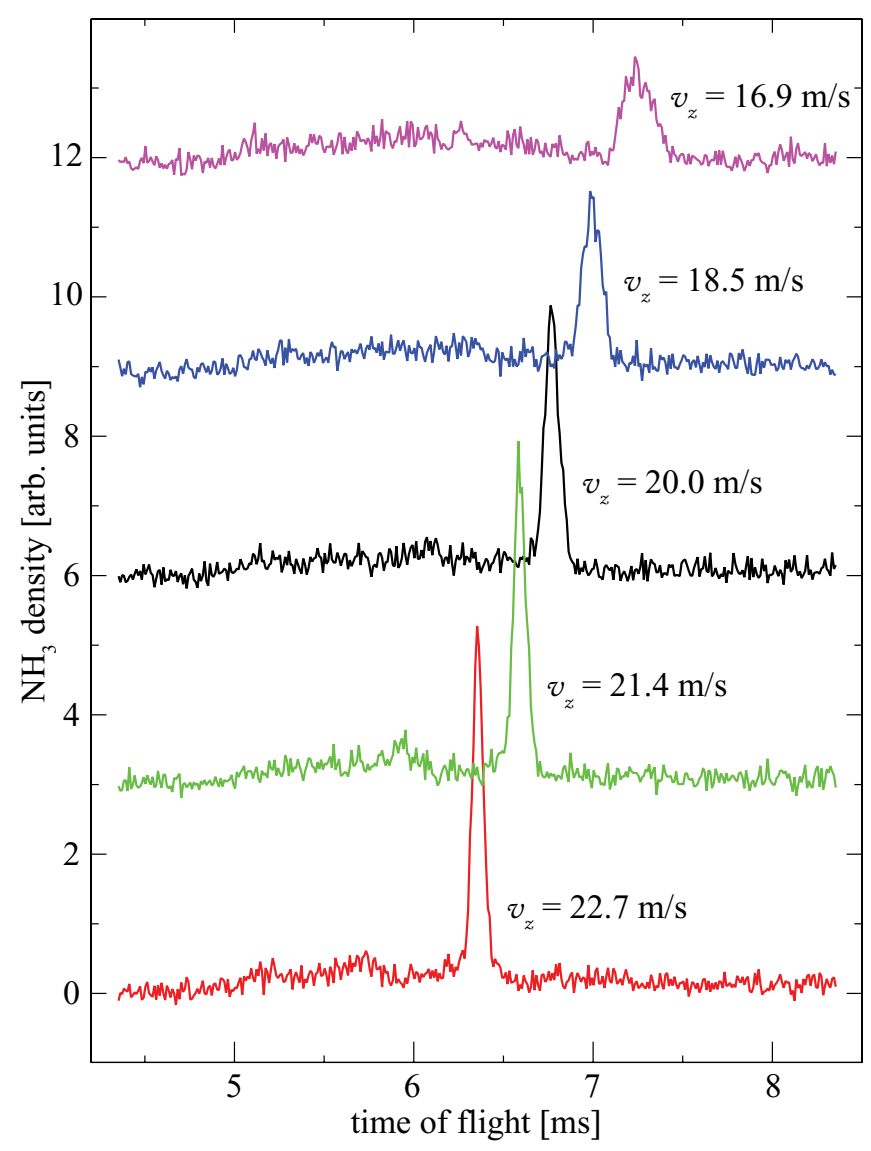

FIG. 3. (Color online) Density of ammonia molecules behind the microwave cavity as a function of time for two different acceleration, one guiding, and two deceleration sequences (curves vertically offset for clarity). Ammonia molecules that enter the cavity at $v_{z}=20.0 \mathrm{~m} / \mathrm{s}$ exit at the indicated velocity. 
packet, but with a broad longitudinal velocity distribution, is created in the detection region; a narrow longitudinal velocity distribution, at the cost of an extended spatial distribution, can be created if the parameters are selected to perform only a quarter of a full rotation in phase space in the microwave cavity. These bunching and longitudinal cooling schemes in a microwave cavity for molecules in high-field-seeking states are similar to schemes demonstrated before with switched electric fields for molecules in low-field-seeking states [22].

The results of microwave acceleration and deceleration experiments are shown in Fig. 3. For these measurements, the microwaves are again switched on and off such that the synchronous molecule experiences two thirds of the potential wells, but at intervals that are no longer distributed symmetrically around the electric field maxima. In addition, the corresponding time intervals are no longer equidistant, due to the nonzero acceleration. The microwave fields are switched such that a synchronous ammonia molecule that enters the microwave cavity at an initial velocity of $v_{z}=20.0 \mathrm{~m} / \mathrm{s}$ gains or loses a fixed amount of kinetic energy $h \times 100 \mathrm{MHz}$ $(h \times 201 \mathrm{MHz})$ per microwave deceleration stage, where $h$ is Planck's constant. These molecules then exit the cavity at velocities of $21.4 \mathrm{~m} / \mathrm{s}(22.7 \mathrm{~m} / \mathrm{s})$ or $18.5 \mathrm{~m} / \mathrm{s}(16.9 \mathrm{~m} / \mathrm{s})$, respectively, as indicated next to the observed arrival time distributions. The time-of-flight distribution obtained when guiding molecules at a constant velocity of $20.0 \mathrm{~m} / \mathrm{s}$ is shown as the middle (black) trace in Fig. 3. Although the longitudinal acceptance of the microwave decelerator gets smaller with increasing acceleration and deceleration rates, the peak intensity in the arrival time distributions is seen to increase slightly for the accelerated beams. This is explained by the improved transverse and longitudinal spatial focusing in the detection region in this case.

\section{CONCLUSIONS}

In conclusion, a prototype microwave decelerator, enabling excellent control over the six-dimensional phase-space distribution of an ensemble of molecules in high-field-seeking states, has been experimentally demonstrated. This microwave decelerator can be used, for instance, to prepare samples of ground-state molecules for subsequent spectroscopy and scattering experiments or to load them into a (microwave) trap.

\section{ACKNOWLEDGMENTS}

We acknowledge help with the design of the cooled microwave cavity from $\mathrm{H}$. Haak and useful discussions with B. G. Sartakov. This work was supported by the ERC2009-AdG program under Grant No. 247142-MolChip. W. J. gratefully acknowledges the support of the Alexander von Humboldt Foundation, and M.S. the support of the Fonds der Chemischen Industrie.
[1] S. Y. T. van de Meerakker, H. L. Bethlem, and G. Meijer, Nature Phys. 4, 595 (2008), and references therein.

[2] S. D. Hogan, M. Motsch, and F. Merkt, Phys. Chem. Chem. Phys. 13, 18705 (2011), and references therein.

[3] L. Scharfenberg et al., Phys. Chem. Chem. Phys. 12, 10660 (2010).

[4] H. L. Bethlem, M. Kajita, B. Sartakov, G. Meijer, and W. Ubachs, Eur. Phys. J. Spec. Topics 163, 55 (2008).

[5] S. Y. T. van de Meerakker, N. Vanhaecke, M. P. J. van der Loo, G. C. Groenenboom, and G. Meijer, Phys. Rev. Lett. 95, 013003 (2005).

[6] H. L. Bethlem, G. Berden, and G. Meijer, Phys. Rev. Lett. 83, 1558 (1999).

[7] N. Vanhaecke, U. Meier, M. Andrist, B. H. Meier, and F. Merkt, Phys. Rev. A 75, 031402(R) (2007).

[8] T. Junglen, T. Rieger, S. A. Rangwala, P. W. H. Pinkse, and G. Rempe, Phys. Rev. Lett. 92, 223001 (2004).

[9] F. Filsinger, U. Erlekam, G. von Helden, J. Küpper, and G. Meijer, Phys. Rev. Lett. 100, 133003 (2008).

[10] M. R. Tarbutt and E. A. Hinds, New J. Phys. 10, 073011 (2008).
[11] H. L. Bethlem, A. J. A. van Roij, R. T. Jongma, and G. Meijer, Phys. Rev. Lett. 88, 133003 (2002).

[12] J. van Veldhoven, H. L. Bethlem, and G. Meijer, Phys. Rev. Lett. 94, 083001 (2005).

[13] R. Grimm, M. Weidemüller, and Y. Ovchinnikov, Adv. At. Mol. Phys. 42, 95 (2000).

[14] R. Fulton, A. I. Bishop, M. N. Shneider, and P. F. Barker, Nat. Phys. 2, 465 (2006).

[15] S. Kuma and T. Momose, New J. Phys. 11, 055023 (2009).

[16] D. DeMille, D. R. Glenn, and J. Petricka, Eur. Phys. J. D 31, 375 (2004).

[17] K. Enomoto and T. Momose, Phys. Rev. A 72, 061403(R) (2005).

[18] R. Hill and T. Gallagher, Phys. Rev. A 12, 451 (1975).

[19] H. Odashima, S. Merz, K. Enomoto, M. Schnell, and G. Meijer, Phys. Rev. Lett. 104, 253001 (2010).

[20] H. L. Bethlem, F. M. H. Crompvoets, R. T. Jongma, S. Y. T. van de Meerakker, and G. Meijer, Phys. Rev. A 65, 053416 (2002).

[21] H. L. Bethlem, G. Berden, A. J. A. van Roij, F. M. H. Crompvoets, and G. Meijer, Phys. Rev. Lett. 84, 5744 (2000).

[22] F. M. H. Crompvoets, R. T. Jongma, H. L. Bethlem, A. J. A. van Roij, and G. Meijer, Phys. Rev. Lett. 89, 093004 (2002). 